\title{
Original
}

\section{Myofibrous Organization in Human Abdominal Muscles}

\author{
Junji ITo and Seiichiro INOKUCHI
}

\begin{abstract}
Myofibrous organization was studied in the abdominal muscles (rectus abdominis: RA, obliquus externus: OE, obliquus internus: OI, transversus abdominis: TA and quadratus lumborum: $\mathrm{QL}$ ) from 14 cadavers (8 males and 6 females, aged 46-90 years) to analyze functions of the abdominal muscles. In RA, the fiber size was approximately equal to that of $\mathrm{OE}$ and $\mathrm{TA}$ and belonged in the moderate muscle fiber group of human muscle. The fiber density in RA was the lowest among the abdominal muscles. In the lateral abdominal muscles, OI, which had the greatest thickness in the muscle layer and the largest muscle fibers of the three muscles, was most predominant, and was followed by OE and TA. QL had the largest muscle fibers among the abdominal muscles which belong in the group of relatively large human muscles. Based on the features of the myofibrous organization, these results suggested functional differences among the abdominal muscles, especially between $\mathrm{OE}$ and $\mathrm{OI}$.
\end{abstract}

Key words: myofibrous organization, human abdominal muscles

\section{Introduction}

Skeletal muscle is an active organ for physical movement and its morphological conditions including attachment of the muscle to bone and the muscle form determine its movement. Skeletal muscle consists of many muscle fibers that have different physiological properties and the characteristics of the fiber composition are presumably related to their functional features. In histological study, it has been noted that muscles that have powerful contraction and rough action, have large muscle fibers, and muscles that act continuously and delicately have small fibers. ${ }^{1)}$ In myofibrous organization, differences in the numbers and sizes of muscle fibers in each muscle are caused by the differences in the muscle activity. Accordingly, analysis of the myofibrous organization is useful to explain muscle function. The muscles in the abdominal wall support and protect the abdominal organs, regulate intraabdominal pressure, are related to respiration, and control movement of the trunk (flexion and rotation). The abdominal muscles consist of the anterior: rectus abdominis (RA), lateral: obliquus externus (OE), obliquus internus (OI) and transversus abdominis (TA) and posterior muscle: quadratus lumborum (QL). Because the anatomical arrangements are remarkably different, functional differences are expected among the abdominal muscles. Histological reports of abdominal muscles are scarce, except for the histochemical study by Häggmark and Thorstensson. ${ }^{2)}$ In the present study, the myofibrous organization of the abdominal muscles: RA, OE, OI, TA and QL, was analyzed histologically and the results were compared with those of the other human muscles to help explain functional feature of the abdominal

Department of Anatomy, Showa University School of Medicine, 1-5-8 Hatanodai, Shinagawa-ku, Tokyo 142, Japan. 
muscles.

\section{Materials and Methods}

Muscle samples were obtained from 14 cadavers ( 8 males aged 46-74 years and 6 females aged 46-90 years), fixed by injection of $10 \%$ formalin, in the anatomical laboratory of Showa University. Muscle specimens were removed from the right abdominal muscle: rectus abdominis (RA), obliquus externus (OE), obliquus internus (OI), transversus abdominis (TA) and quadratus lumborum (QL), at the level of navel. Muscle slices were embedded in celloidin and $20 \mu \mathrm{m}$ sections were cut and stained with haematoxylin and eosin. On these sections, the myofibrous organizations: thickness of the muscle layer (OE, OI and TA), cross-sectional area and total number of muscle fibers (RA and QL) in the muscle belly, number of muscle fibers per $\mathrm{mm}^{2}$, mean fiber size and fiber density were measured. To analyze myofibrous organization, 100 measuring points were selected at random in the cross section by a sampling method. To estimate the number of muscle fibers per $\mathrm{mm}^{2}$, the fibers in $0.01 \mathrm{~mm}^{2}$ were counted at each point and then added. To measure muscle fiber size, 200 fibers were traced at a magnification of 400 with a microscopic image system. Cross sectional area of fibers (fiber size) was then measured with a Wacom Digitizer (WT 4400SE, CPU: NEC 9801). Muscle fiber density was estimated by multiplying fiber size by number of fibers per $\mathrm{mm}^{2}$. These results were compared with those of the other human muscles that had been measured by the same methods in our laboratory: trapezius ( $\mathrm{Tr}$ ), rhomboideus major (RM), serratus anterior (SA), ${ }^{3)}$ iliacus (Il), psoas major (PM), ${ }^{4)}$ gastrocnemius laterale (GL) and mediale (GM) and soleus (Sl).5)

\section{Results}

Thickness of the muscle layers was measured in OE, OI and TA, and the results are summarized in Table 1. The thickness was greatest in OI, and least in TA. In each layer, the thickness was greater in males than in females. The thickness of lateral abdominal muscles was compared with that of other human muscles (SA, Tr and RM) in Fig. 1. OE, a relatively thin muscle (about $3.0 \mathrm{~mm}$ ), was about equal to the cranial part of the Tr. The moderate thickness of OI (about $4.0 \mathrm{~mm}$ ) was about equal to the caudal part of the Tr. TA was the thinnest (about $2.0 \mathrm{~mm}$ ) of the muscles observed in this study.

Cross-sectional area and total number of muscle fibers were measured in muscle bellies of RA and QL (Tables 2 and 3) and compared with those of PM, Il, GL, GM and SI (Figs.

Table 1. Thickness of muscle layer $(\mathrm{mm})$ in lateral abdominal muscles. Total mean value in all subjects, mean and ranges (maximum-minimum) in each sex shown. These expressions are similar in all tables.

\begin{tabular}{lccc} 
& Total mean & $\begin{array}{c}\text { Male } \\
\text { (max.-min.) }\end{array}$ & $\begin{array}{c}\text { Female } \\
\text { (max.-min.) }\end{array}$ \\
Obliquus externus & 3.0 & 3.3 & $-\frac{2.5}{(6.6-0.8)}$ \\
Obliquus internus & 4.2 & 4.3 & 4.0 \\
Transversus abdominis & & $(7.1-3.1)$ & $(9.3-2.4)$ \\
& 2.2 & 2.4 & 1.9 \\
\hline
\end{tabular}




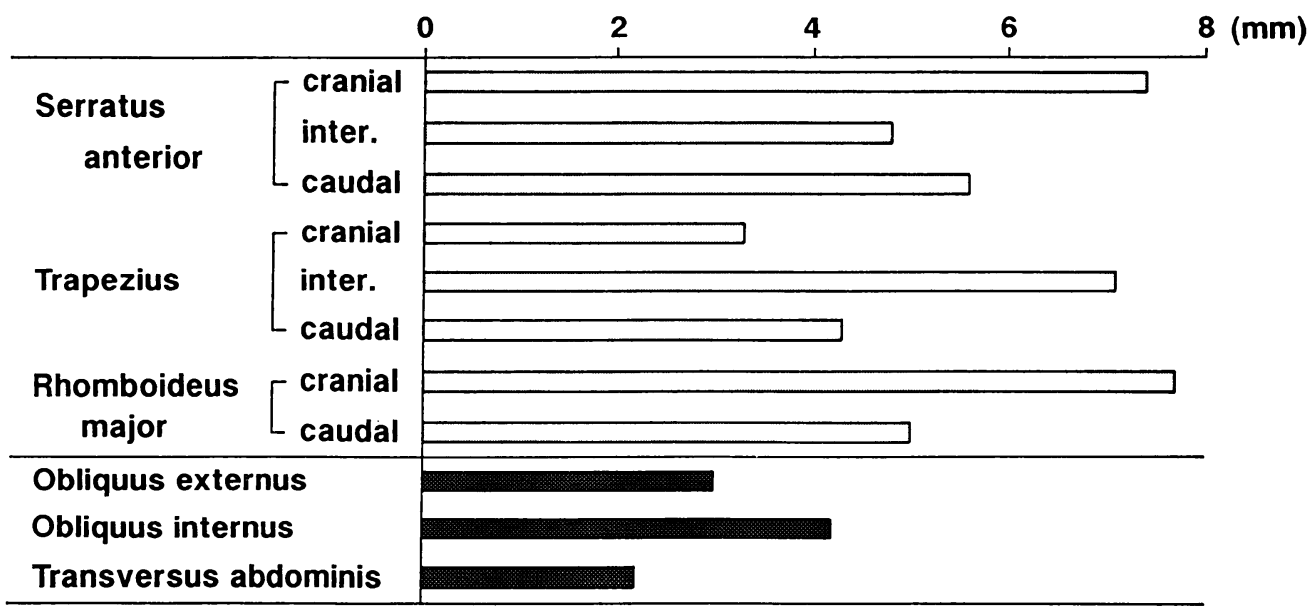

Fig. 1. Comparison of muscle layer thickness in the lateral abdominal muscles ( $\mathrm{m}$. obliquus externus, $\mathrm{m}$. obliquus internus and $\mathrm{m}$. transversus abdominis) with serratus anterior (cranial, intermediate and caudal part), trapezius (cranial, intermediate and caudal part) and rhomboideus major (cranial and caudal part). Values for each muscle are mean of all subjects, and these expressions are similar in the following figures.

Table 2. Cross-sectional area of the muscle belly $\left(\mathrm{mm}^{2}\right)$ in $\mathrm{m}$. rectus abdominis and $\mathrm{m}$. quadratus lumborum.

\begin{tabular}{lcccc} 
& Total mean & $\begin{array}{c}\text { Male } \\
(\max .-\min .)\end{array}$ & $\begin{array}{c}\text { Female } \\
\text { (max.-min.) }\end{array}$ \\
\hline Rectus abdominis & 192.0 & 215.0 & 161.9 \\
Quadratus lumborum & 213.7 & $(283.3-173.4)$ & $(203.2-95.1)$ \\
& & 253.0 & 161.9 \\
& & $(214.0-280.0)$ & $(244.4-111.4)$ \\
\hline
\end{tabular}

Table 3. Total number of muscle fibers in cross section in $\mathrm{m}$. rectus abdominis and $\mathrm{m}$. quadratus lumborum. $(\times 1000)$.

\begin{tabular}{|c|c|c|c|}
\hline & Total mean & $\begin{array}{c}\text { Male } \\
(\max .-\min .)\end{array}$ & $\begin{array}{c}\text { Female } \\
(\max .-\min )\end{array}$ \\
\hline Rectus abdominis & 159.6 & $\begin{array}{c}177.9 \\
(144.4-132.4)\end{array}$ & $\begin{array}{c}135.2 \\
(181.4-83.7)\end{array}$ \\
\hline Quadratus lumborum & 143.9 & $\begin{array}{c}179.6 \\
(230.0-150.2)\end{array}$ & $\begin{array}{c}99.4 \\
(141.8-38.7)\end{array}$ \\
\hline
\end{tabular}

2 and 3). Cross-sectional area in QL was slightly greater than that of RA and both were approximately equal to PM (about $200 \mathrm{~mm}^{2}$ ) (Fig. 2). Conversely, the total number of muscle fibers of RA exceeded that of QL. The total numbers in both muscles were considerably less than those in other human muscles, which exceeded 200,000 (Fig. 3). In both categories, both muscles in males exceeded those in females.

Number of muscle fibers per $\mathrm{mm}^{2}$ in the abdominal muscles is shown in Table 4 . The number of fibers was the largest in OE and smallest in QL. The number in TA was similar 


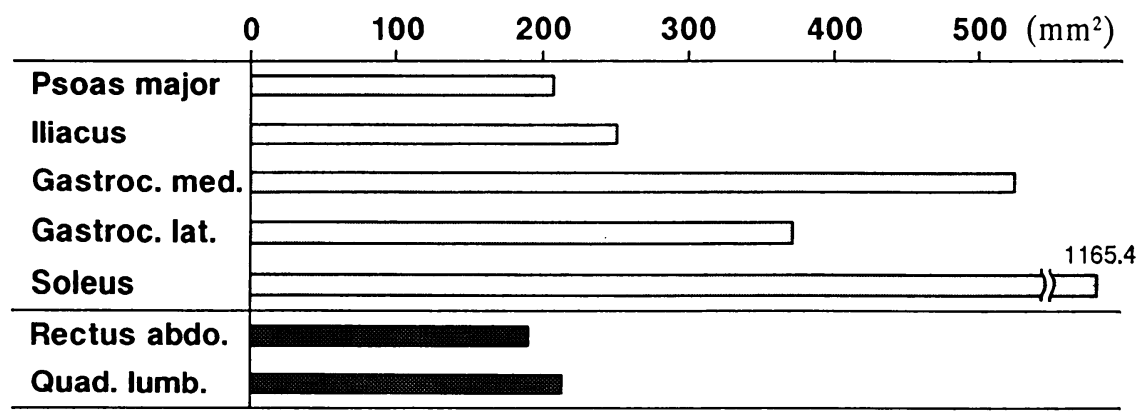

Fig. 2. Comparison of the cross-sectional areas of the muscle bellies in $\mathrm{m}$. rectus abdominis and $\mathrm{m}$. quadratus lumborum with those in $\mathrm{m}$. iliopsoas (m. psoas major and $\mathrm{m}$. iliacus) and triceps surae ( $\mathrm{m}$. gastrocnemius lateral head and medial head and $\mathrm{m}$. soleus).

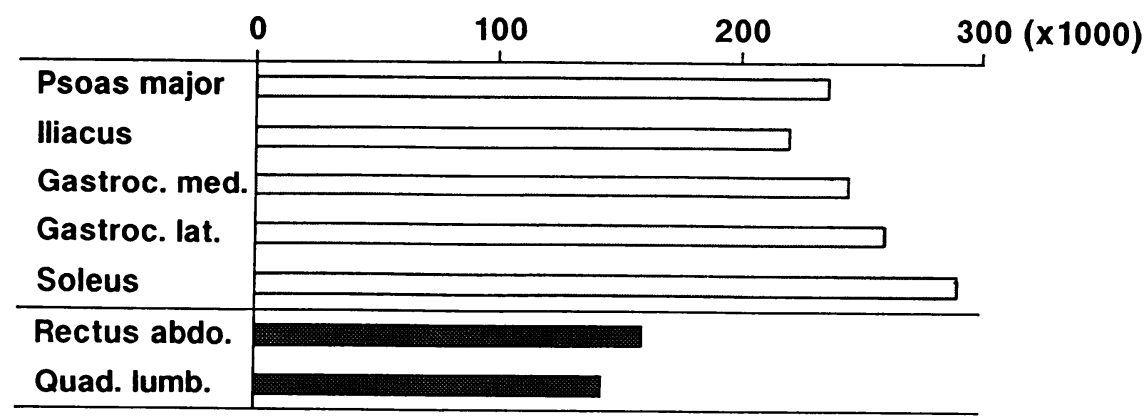

Fig. 3. Comparison of the total number of muscle fibers in the cross sections in $\mathrm{m}$. rectus abdominis and $\mathrm{m}$. quadratus lumborum with those in $\mathrm{m}$. iliopsoas and $\mathrm{m}$. triceps surae.

Table 4. Number of muscle fibers per $\mathrm{mm}^{2}$ in abdominal muscles.

\begin{tabular}{lcccc}
\hline & Total mean & $\begin{array}{c}\text { Male } \\
(\text { max.-min. })\end{array}$ & $\begin{array}{c}\text { Female } \\
(\text { max.-min. })\end{array}$ \\
\hline Rectus abdominis & 851 & $\begin{array}{c}840 \\
(1292-636)\end{array}$ & $\begin{array}{c}867 \\
(1346-613)\end{array}$ \\
Obliquus externus & 1189 & 1091 & 1321 \\
Obliquus internus & 880 & $(1686-748)$ & $(1707-879)$ \\
Transversus abdominis & 1134 & $\begin{array}{c}834 \\
(1037-647)\end{array}$ & $\begin{array}{c}941 \\
(1329-573)\end{array}$ \\
Quadratus lumborum & & $(1409-744)$ & 1137 \\
& 663 & 720 & $(1749-691)$ \\
& & $(902-497)$ & $(758-334)$ \\
\hline
\end{tabular}




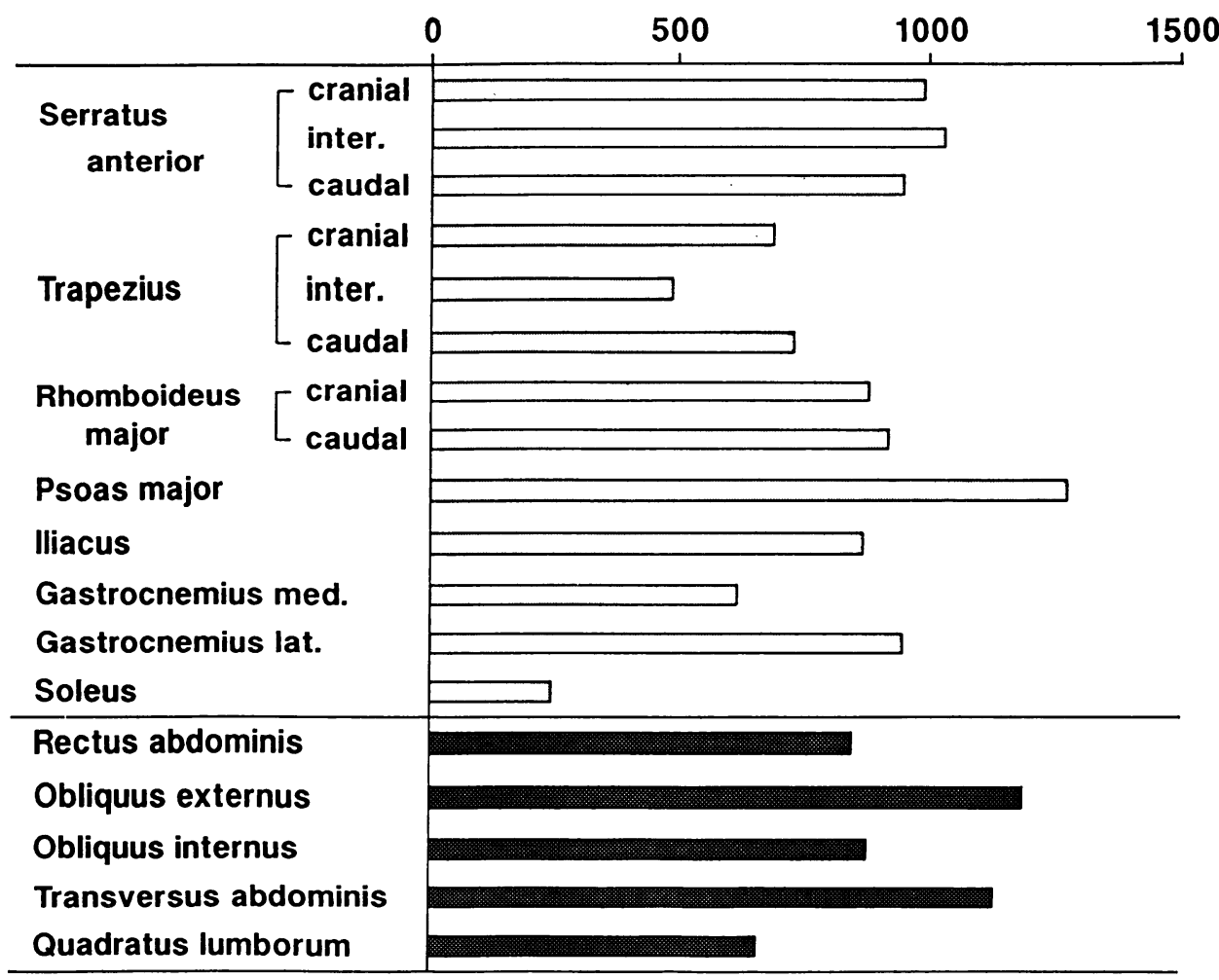

Fig. 4. Comparison of the number of muscle fibers per $\mathrm{mm}^{2}$ in abdominal muscles with those in other human muscles.

to that in OE and those in OI and RA were intermediate between OE and QL. The fiber number per $\mathrm{mm}^{2}$ in RA, OE and $\mathrm{OI}$ in females exceeded that in males, but vice versa in QL. In TA, the numbers were similar in both sexes. The number of muscle fibers in the abdominal muscles was compared with that in other human muscles (Fig. 4). OE and TA had more (about 1100) than most other muscles, OI and RA had moderate numbers (about 850) about equal to the cranial part of RM and Il, and QL had the least (below 700) about equal to the cranial part of $\mathrm{Tr}$ and GM.

The mean size of muscle fibers in abdominal muscles is shown in Table 5. The largest muscle fiber was found in QL, and the size decreased in the order of OI, RA, OE and TA. In females, the TA fibers were larger than the RA and OE fibers. The size in males was larger than that of females, except for $\mathrm{QL}$, and the sex differences were large in RA, OE and OI. Mean size of the abdominal muscles was compared with that of other human muscles (Fig. 5). QL and OI, the largest (about $1100 \mu \mathrm{m}^{2}$ ) of the abdominal muscles were about equal to $\mathrm{Tr}$ and GM. Three other muscles (OE, TA and RA) were almost equal to SA and RM and belonged in the moderate muscle groups (about $800 \mu \mathrm{m}^{2}$ ).

The lateral abdominal muscles had higher muscle fiber density $(87.8 \%$ for OI, $85.8 \%$ for OE and $84.0 \%$ for TA) than QL (74.8\%) and RA (66.9\%) (Table 6). In all muscles, the densities in males were higher than those in females. 


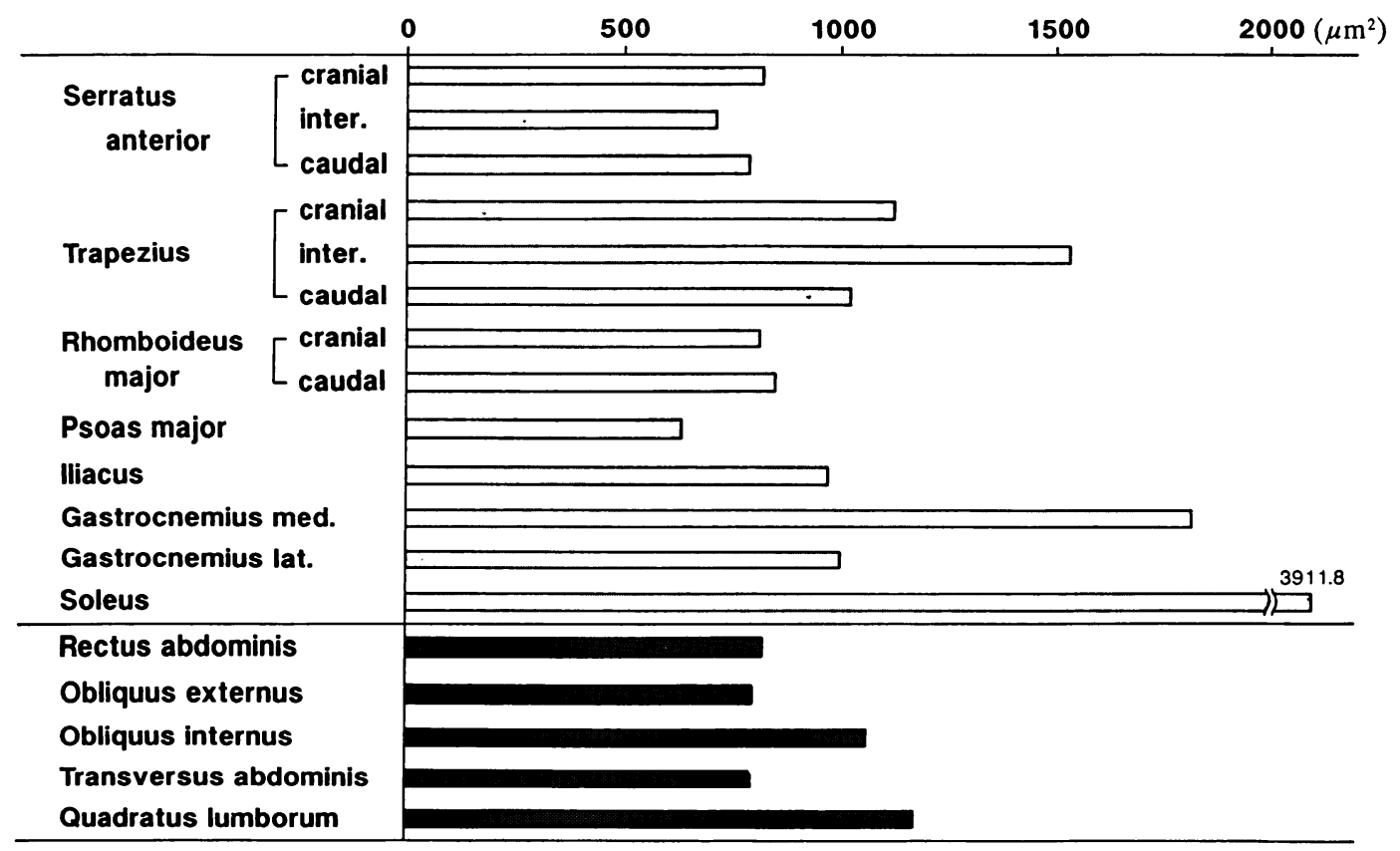

Fig. 5. Comparison of the mean fiber size in abdominal muscles with that in other human muscles.

Table 5. Mean fiber sizes $\left(\mu \mathrm{m}^{2}\right)$ of abdominal muscles.

\begin{tabular}{|c|c|c|c|}
\hline & Total mean & $\begin{array}{c}\text { Male } \\
\text { (max.-min.) }\end{array}$ & $\begin{array}{c}\text { Female } \\
(\max .-\min .)\end{array}$ \\
\hline Rectus abdominis & 825.4 & $\begin{array}{c}899.7 \\
(1157.9-647.2)\end{array}$ & $\begin{array}{c}726.3 \\
(1023.9-419.8)\end{array}$ \\
\hline Obliquus externus & 800.1 & $\begin{array}{c}896.0 \\
(1346.3-559.3)\end{array}$ & $\begin{array}{c}672.2 \\
(1096.7-430.9)\end{array}$ \\
\hline Obliquus internus & 1061.5 & $\begin{array}{c}1147.4 \\
(1447.2-848.2)\end{array}$ & $\begin{array}{c}947.0 \\
(1629.3-551.6)\end{array}$ \\
\hline Transversus abdominis & 796.3 & $\begin{array}{c}808.4 \\
(1140.4-598.3)\end{array}$ & $\begin{array}{c}780.3 \\
(1163.9-426.0)\end{array}$ \\
\hline Quadratus lumborum & 1178.8 & $\begin{array}{c}1173.2 \\
(1403.2-992.1)\end{array}$ & $\begin{array}{c}1186.4 \\
(2222.7-845.9)\end{array}$ \\
\hline
\end{tabular}

\section{Discussion}

From the results of this study, features of the myofibrous organization of abdominal muscles are summarized as follows: The mean size of RA muscle fibers was approximately equal to $\mathrm{OE}$ and TA and belonged in the moderate fiber group in human muscles (Fig. 5), although the number of muscle fibers per $\mathrm{mm}^{2}$ was less than in those muscles (Fig. 4). The density of muscle fibers was lowest in the abdominal muscles (Table 6). The total number of muscle fibers and the cross-sectional area were approximately equal to those of QL, but they were much smaller than iliopsoas and triceps surae (Figs. 2 and 3). In the lateral ab- 
Table 6. Muscle fiber density (\%) in abdominal muscles.

\begin{tabular}{|c|c|c|c|}
\hline & Total mean & $\begin{array}{c}\text { Male } \\
(\max .-\min )\end{array}$ & $\begin{array}{c}\text { Female } \\
(\max .- \text { min. })\end{array}$ \\
\hline Rectus abdominis & 66.9 & $\begin{array}{c}73.0 \\
(83.6-64.6)\end{array}$ & $\begin{array}{c}58.9 \\
(63.9-51.6)\end{array}$ \\
\hline Obliquus externus & 85.8 & $\begin{array}{c}90.3 \\
(94.9-84.9)\end{array}$ & $\begin{array}{c}80.5 \\
(96.4-64.7)\end{array}$ \\
\hline Obliquus internus & 87.8 & $\begin{array}{c}93.2 \\
(98.4-85.8)\end{array}$ & $\begin{array}{c}80.6 \\
(93.4-69.6)\end{array}$ \\
\hline Transversus abdominis & 84.0 & $\begin{array}{c}88.2 \\
(93.1-84.1)\end{array}$ & $\begin{array}{c}78.4 \\
(89.6-67.8)\end{array}$ \\
\hline Quadratus lumborum & 74.8 & $\begin{array}{c}83.1 \\
(93.7-69.3)\end{array}$ & $\begin{array}{c}63.8 \\
(74.2-55.1)\end{array}$ \\
\hline
\end{tabular}

dominal muscles, OI was most predominant in thickness of the muscle layer (Table 1), muscle fiber size (Table 5), belonging in the relatively large fiber group in human muscles, and fiber density (Table 6). Between OE and TA, myofibrous organization, including muscle layer thickness, was similar in each element, but OE was slightly more developed than TA. The mean fiber size in QL was largest in the abdominal muscles and was similar to those in Tr and GM (Fig. 5), which belong in the relatively large fiber group, but the number of muscle fibers per $\mathrm{mm}^{2}$ was smallest in the abdominal muscles (Fig. 4). Total fiber number and cross-sectional area were approximately equal to RA. From the point of view that muscle characteristics are related to their functions, the differences in myofibrous organization of the human abdominal muscles reveal features and action differences of these muscles. In addition, the differences between $\mathrm{OE}$ and $\mathrm{OI}$ were made especially clear in this study. A histochemical study ${ }^{2)}$ concluded that the abdominal muscles had similar functional capacity. Electromyographic studies $^{(6-8)}$ reported functional differences between the anterior abdominal muscles (RA) and lateral muscles (OE, OI and TA), but did not mention the differences in the lateral abdominal muscles. The results of the present study suggest functional differences among the lateral abdominal muscles.

In his histological study of human skeletal muscles, Kohashi noted that muscles whose contractions were powerful and non-sustained had large muscle fibers, whereas the muscles that had a continuous or delicate activity possessed small fibers. ${ }^{11}$ Fiber size is increased by endurance training. ${ }^{9,10)}$ Among sportsman, especially weight lifters, body builders and boxers, fiber size was about two or three times that in non-sportsmen ${ }^{11}$ and the subjects in the present study. Fiber size increases with increase in load and in muscle movement. In the present study, QL had the largest fiber size among the abdominal muscles (Fig. 5) and was similar to Tr and GM and the upper and lower extremity muscles. ${ }^{12,13)}$ In OI, the muscle fiber size was similar to that in QL and greater than those in anterior and other lateral abdominal muscles. These facts show that, in the two muscles, OI and QL, the load during muscle movement was greater than for other muscles.

Functions of the abdominal muscles are roughly divided into the following: 1) support and protect the abdominal organs, 2) participate in respiration, 3) regulate intra-abdominal pressure, and 4) flexion (lateral and ventral) and rotation of the trunk. The expected actions 
of the abdominal muscles as determined from their anatomical arrangement are as follows: OE and OI participate in all functions, 1), 2), 3) and 4), TA in 1), 2) and 3), RA in 4) when it is ventral flexion and QL in 4) when it is lateral flexion. ${ }^{14}$

From the anatomical arrangements and features of the myofibrous organization of the abdominal muscles, the following can be considered: The differences in the myofibrous organization between $\mathrm{OE}$ and $\mathrm{OI}$ show the active differences in the rotation. That is, during this movement, OI on the direction side moves with $\mathrm{OE}$ on the opposite side. It is morphologically very probable that the load on OI, which takes its origin from the iliac crest in the direction of rotation, is heavier than that on OE. In the support and protection of the abdominal organs, TA which forms a broad band running transversely around the abdominal cavity, is presumed to be the most effective. These active features in TA correspond to the facts that its muscle fiber was smallest among the abdominal muscles and no sex differences were observed in the muscle fiber size. Features of the myofibrous organization in QL show that its load during lateral flexion of the trunk is greater than that of the other anterior and lateral abdominal muscles.

The functions of skeletal muscles can be analyzed by histochemically classifying the muscle fibers according to their physiological properties and studying the composition of the fiber types composing each muscle. The muscle fibers can then classified into Type I and Type II based on the difference in myosin ATPase and the Type II fiber is further subdivided into Type IIA and IIB. ${ }^{15)}$ Type I, IIA and IIB fibers are also called, respectively, slowtwitch-oxidative (SO), fast-twich-oxidative-glycolytic (FOG) and fast-twitch-glycolytic (FG) fiber, ${ }^{16)}$ or red, intermediate and white muscle fiber. ${ }^{1 i)}$ Muscles with tonic functions contain a high ratio of Type I fibers (red muscle type), and muscles with phasic functions contain a high ratio of Type II fibers (white muscle type). ${ }^{18)}$ Johnson et al. ${ }^{19)}$ reported that the Type I fiber ratio was $87 \%$ for soleus, which functions as a tonic muscle, and $38 \%$ for vastus lateralis, a phasic muscle. Häggmark and Thorstensson ${ }^{2)}$ reported the Type II fiber ratio was 55 to $58 \%$ in the human abdominal muscles with only slight differences between the muscles. This value is intermediate between those of soleus and vastus lateralis. This ratio is clearly higher than the ratio in the extremity muscles. The Type I fiber ratio was 33 to $77 \%$ for OE, OI and RA, showing considerable differences between individuals and some muscles even had ratios of less than $50 \%$. On the contrary, all specimens of TA had ratios of $50 \%$ or more, with smaller differences between the individuals. These findings lead to the conclusion that functions of the abdominal muscles, especially TA, are relatively tonic. These conclusions suggest that support and protection of the abdominal organs constitute the tonic function and the trunk movement is the phasic one. It is reasonable, too, consistent with the above discussion, that features of the myofibrous organization differ between OE, OI and TA.

In the present study, sex differences in each element of the myofibrous organization were observed and some differences might be caused by aging. But in the human skeletal muscles which were studied in our laboratory, the muscles in males generally had a tendency to be larger than those in females.

\section{References}

1) Kohashi Y: Histologische Untersuchungen der verschiedenen Skelettmuskeln beim Menschen. I. Untersuchungen beim Erwachsenen. Folia Anat Jpn, 15: 175-188 (1937)

2) Häggmark $T$ and Thorstensson A: Fibre types in human abdominal muscles. Acta Physiol Scand, 
107: 319-325 (1979)

3) Ito R: Study on the myofibrous organization of human spinohumeral muscle. J Showa Med Assoc, 43: 43-54 (1983) (in Japanese)

4) Hasegawa $\mathbf{M}$ : On the myofibrous organization of human iliopsoas muscle (psoas major muscle and iliacus muscle). J Showa Med Assoc, 47: 833-842 (1987) (in Japanese)

5) Kataoka J: Comparative studies on muscle structure of the $\mathrm{m}$. triceps surae and $\mathrm{m}$. plantaris in man and monkey. J Showa Med Assoc, 47: 851-861 (1987) (in Japanese)

6) Floyd WF and Silver PHS: Electromyographic study of patterns of activity of the anterior abdominal wall muscles in man. J Anat, 84: 132-145 (1950)

7) Waters RL and Morris JM: Electrical activity of muscles of the trunk during walking. J Anat, 111: 191-199 (1972)

8) Furlani $\mathbf{J}$ and Bankoff ADP: Electromyographic analysis of the rectus abdominis and external oblique muscles during exercises in lateral decubence, sitting and kneeling positions. Electromyogr Clin Neurophysiol, 27: 265-272 (1987)

9) Klausen K, Andersen LB and Pelle I: Adaptive changes in work capacity, skeletal muscle capillarization and enzyme levels during training and detraining. Acta Physiol Scand, 113: 9-16 (1981)

10) Ito K: Effects of endurance training on histochemical properties of rat skeletal muscle. Jpn J Phys Fitness Sports Med, 30: 157-166 (1981) (in Japanese)

11) Inokuchi $S$, Iwamoto $S$, Ishikawa $H$ and Higashi $Y$ : Myofibrous organization of the $m$. rectus abdominis from 3 sportsmen (weight-lifting, boxing and body-building). Jpn J Phys Fitness Sports Med, 29: 45-51 (1980) (in Japanese)

12) Kimura T: Myofibrous development of the lower extremity muscles of rhesus monkey (Macaca mulatta). J Showa Med Assoc, 38: 593-603 (1978) (in Japanese)

13) Teratani, $T$, Ito $J$, Ito $R$, Inokuchi $S$ and Matuyama $Y$ : Studies on the myofibrous organization of upper extremity muscles in man-Comparison of the function of upper extremity in man and monkey. J Showa Med Assoc, 45: 61-70 (1985) (in Japanese)

14) Duvall EN: Kinesiology. The Anatomy of Motion. Prentice-Hall Inc., Englewood Cliffs, N.J., pp 150-162 (1959)

15) Peter JB, Barnard J, Edberton VR, Gillespie CA and Stempel KE: Metabolic profiles of three fiber types of skeletal muscle in guinea pigs and rabbits. Biochemistry, 11: 2627-2633 (1972)

16) Brook MH and Kaiser KK: Muscle fiber types: How many and what kind? Arch Neurol, 23: 369379 (1970)

17) Ogata T: A histochemical study of the red and white muscle fibers. Part I. Activity of the succinoxydase system in muscle fibers. Acta Med Okayama, 12: 216-227 (1958)

18) Gollnick PD, Sjodin B, Karlsson J, Janssom E and Saltin B: Human soleus muscle: A comparison of fiber composition and enzyme activities with other leg muscles. Pflugers Arch, 348: 247-255 (1974)

19) Johnson MA, Polgar J, Weightman D and Appleton D: Date on the distribution of fibre types in thirty-six human muscles. An autopsy study. J Neurol Sci, 18: 111-129 (1973)

[Received September 1, 1989: Accepted October 20, 1989] 